\title{
Injeção epidural preventiva de xilazina ou amitraz, em eqüinos: efeito antinociceptivo
}

\author{
Pre-emptive epidural injection of xylazine or amitraz, in horses: antinociceptive effect
}

\author{
Erica Cristina Bueno do Prado Guirro ${ }^{\text {I }}$ Guilherme Roberto Sobrinho ${ }^{\text {II }}$ \\ Isabella Maria Marchesini Ferreira ${ }^{\mathrm{III}}$ Carlos Augusto Araújo Valadão ${ }^{\mathrm{IV}}$
}

\section{RESUMO}

Os agonistas de receptores adrenérgicos $\alpha^{2}$ geram efeito antinociceptivo após administração epidural e o amitraz possui atividade intrínseca sobre tais receptores. Assim, avaliouse o efeito antinociceptivo promovido pela injeção epidural preventiva de $0,17 \mathrm{mg} \mathrm{kg}^{-1}$ de xilazina (GX) ou $0,1 \mathrm{mg} \mathrm{kg}^{-1}$ de amitraz diluído em emulsão lipídica (GA) durante 24 horas, em doze eqüinos submetidos a modelo incisional de dor inflamatória e avaliados com filamentos de von Frey quanto ao limiar nociceptivo à estimulação mecânica (LNEM). A xilazina produziu manutenção do LNEM por até 4 horas, enquanto que o amitraz diluído em emulsão lipídica foi capaz de manter o LNEM inalterado por até 24 horas.

Palavras-chave: amitraz, analgesia, epidural, eqüinos, nocicepção, xilazina.

\section{ABSTRACT}

Epidural administration of $\alpha^{2}$ agonists promotes antinociceptive effect and amitraz acts on $\alpha^{2}$ receptors. Antinociceptivo effect caused by pre-emptive epidural injection of $0.17 \mathrm{mg} \mathrm{kg}^{-1}$ xylazine (GX) or $0.1 \mathrm{mg} \mathrm{kg}^{-1}$ amitraz diluted in lipidic emulsion (GA) were evaluated, in 12 horses submitted by incisional model of inflammatory pain and evaluated about nociceptive threshold by mechanical stimulus (NTMS), by von Frey filaments. Xylazine produced maintenance of NTMS for 4 hours, while amitraz diluted in lipidic solution maintained NTMS for 24 hours.

Key words: amitraz, analgesia, epidural, horses, nociception, xylazine.

\section{INTRODUÇÃO}

A dor é definida pela Associação Internacional para o Estudo da Dor como "uma experiência sensitiva e emocional desagradável associada à lesão tecidual real ou potencial" (LEMONICA \& PEREIRA, 1992). A dor pós-incisional caracteriza-se como dor inflamatória (BUERKLE et al., 1999), pois há liberação de mediadores inflamatórios que reduzem o limiar de fibras aferentes, principalmente do tipo $\mathrm{C}$, levando à sensibilização central e seus efeitos deletérios como hiperalgesia e alodinia (WOOLF \& CHONG 1993; MICHAELIS et al., 1996; VALADÃO et al., 2002). Na experimentação animal, sugere-se a substituição dos termos dor e analgesia por nocicepção e antinocicepão, respectivamente (JONES, 1992). PARADA et al., 2003 e CUNHA et al., 2004 propõem o termo hipernocicepção para referir, indistintamente, alodinia, hiperalgesia primária ou secundária.

A presença de dor no período pós-operatório reduz a qualidade de vida do paciente (WU et al., 2003), pois desequilibra a homeostase e gera alterações autonômicas, endócrinas e comportamentais (DANNEMAN, 1997). Portanto, diversas técnicas de analgesia são sugeridas para minimizá-la e a analgesia preventiva é descrita como o uso de analgésicos antes

IPrograma de Pós-graduação em Cirurgia Veterinária, Faculdade de Ciências Agrárias e Veterinárias (FCAV), Universidade Estadual Pauliasta (UNESP), 14884-900, Jaboticabal, SP, Brasil. Universidade Federal do Paraná (UFPR), Campus Palotina, 85950-000, Palotina, PR, Brasil. E-mail: ericaguirro@ufpr.br. Autor para correspondência.

IIAutônoma

IIIMinistério da Agricultura, Pecuária e Abastecimento (MAPA), Iporã, PR, Brasil.

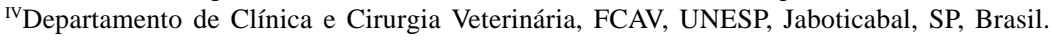


do trauma a fim de minimizar os efeitos da sensibilização central (WOOLF \& BROMLEY, 1999; WARNCKE et al., 2000).

O uso epidural de fármacos antinociceptivos possibilita intervenções urogenitais em eqüinos em estação (SKARDA, 1991; SALMON et al., 1995; DOHERTY et al., 1997), sendo que os anestésicos locais são utilizados, mas geram bloqueio motor e simpático além do sensorial (DE ROSSI et al. 2005). Na busca de mais seletividade por fibras sensoriais, surgem os agonistas $\alpha^{2}$ (LE BLANC \& CARON, 1990; LE BLANC \& EBERHART, 1990; GRUBB et al., 1992).

A aplicação sistêmica de agonistas $\alpha^{2}$ produz sedação, miorrelaxamento e analgesia (BUERKLE et al., 1999; MUIR III et al., 2001) e após administração epidural há antinocicepção mais prolongada e mínimos efeitos colaterais (GRUBB et al., 1992; KEEGAN et al., 1995) devido à redução da dose (LE BLANC \& EBERHART, 1990) e da absorção sistêmica (VALADÃO et al., 1990; DE ROSSI \& GÓMEZ DE SEGURA, 2001).

A xilazina é o agonista $\alpha^{2}$ mais empregado em grandes animais e, em eqüinos, seu uso epidural aumenta a antinocicepção perineal, diminui a ataxia e não interfere nas variáveis cardiorrespiratórias (LE BLANC et al., 1988; KEEGAN et al., 1995; SKARDA \& MUIR III, 1996a; SKARDA \& MUIR III, 1996b; DE ROSSI \& GÓMEZ DE SEGURA, 2001).

O amitraz é um ectoparasiticida que atua em receptores $\alpha^{2}$ centrais e periféricos (HSU et al., 1986; ROBERTS \& ARGENZIO, 1986; HUGNET et al., 1996; LEE et al., 2003) e comparado à xilazina, em eqüinos, produz sedação mais intensa e prolongada, interfere menos na motilidade intestinal e reduz a locomoção (HARKINS et al., 1997; QUEIRÓZ-NETO et al., 2000), sem ser tóxico ao fígado, rins, músculos, sangue ou alterar os níveis séricos de eletrólitos (QUEIRÓZ-NETO, 1997; QUEIRÓZ-NETO et al., 2000). O uso epidural de amitraz em eqüinos gera sedação, miorrelaxamento, alterações cardiorrespiratórias mínimas e antinocicepeção (VALADÃO, 1998).

A maioria dos estudos sistêmicos com amitraz utilizou diluição em dimetilformamida, xileno ou dimetil sulfóxido (HSU et al., 1986; VALADÃO, 1998; QUEIRÓZ-NETO et al., 2000; BALESTRERO, 2001; FARIAS et al., 2002; ALMEIDA et al., 2005). Todavia, a diluição em emulsão lipídica garante maior permanência do fármaco no espaço epidural e minimização dos efeitos colaterais oriundos da absorção sistêmica (FARIAS, 2004; LINARDI, 2004; MENDES, 2004). O uso epidural do amitraz neste veículo não promove sinais neurológicos e nem lesões histopatológicas na medula de cães (FARIAS, 2004).
Os filamentos de von Frey podem ser empregados para avaliar a ação antinociceptiva de determinado fármaco, pois pressionados ao redor do tecido injuriado estabelecem o limiar doloroso a partir do filamento de maior diâmetro incapaz de gerar desconforto (PEDERSEN et al., 1998). Este método já foi utilizado com sucesso em eqüinos (OLESKOVICZ et al., 2001; RÉDUA et al., 2002; MORAES, 2003; GUIRRO, 2005).

Assim, este estudo avaliou a ação antinociceptiva preventiva do amitraz diluído em emulsão lipídica comparado a xilazina, em eqüinos submetidos a um modelo incisional de dor inflamatória.

\section{MATERIAL E MÉTODOS}

Foram utilizados cinco eqüinos machos castrados e sete fêmeas não prenhes e fora de estro, de 3 a 7 anos e peso de $318 \pm 48 \mathrm{~kg}$. O início dos ensaios foi padronizado às $8 \mathrm{~h}$ para evitar variações decorrentes do ciclo circadiano. Para minimizar outras interferências, a avaliação foi feita por um observador “cego" e os animais foram previamente adaptados à rotina experimental.

Após enfaixamento da cauda e tricotomia das regiões sacrococcígea e isquiática dos dois membros pélvicos, desenhou-se uma linha vertical de $10 \mathrm{~cm}$ a partir da tuberosidade isquiática e retângulos a 1, 3 e $5 \mathrm{~cm}$ ao redor com tinta branca hidrossolúvel. Esse foi considerado T-30 e realizou-se a aferição basal do limiar nociceptivo ao estímulo mecânico (LNEM) com os filamentos de von Freya, iniciando-se pelo filamento menos calibroso que foi seqüencialmente substituído pelo filamento imediatamente mais espesso até gerar resposta aversiva, sendo o LNEM estabelecido pelo maior filamento incapaz de gerar resposta adversa. Foi seguida a ordem dorsal, direito, esquerdo e ventral a 1, 3 e $5 \mathrm{~cm}$ da linha central, em ambos os membros pélvicos. Quanto maior a ação antinociceptiva do fármaco, mais calibroso é o filamento necessário para provocar resposta adversa.

Em seguida, infiltrou-se 3mL lidocaína 2\% no espaço subcutâneo de $\mathrm{Co}_{1}-\mathrm{Co}_{2}$ e após 5 minutos perfurou-se a pele com agulha hipodérmica antes da inserção da agulha espinhal de Tuohy até o espaço epidural para permitir a passagem do cateter epidural e introdução cranial por $15 \mathrm{~cm}$ pelo qual se administrou os fármacos: GX (0,17 $\mathrm{mg} \mathrm{kg}^{-1}$ de xilazina) ou GA $(0,1 \mathrm{mg}$ $\mathrm{kg}^{-1}$ de amitraz diluído em emulsão lipídica). A aplicação foi padronizada em 2 minutos e o volume final (mL) foi ajustado com $\mathrm{NaCl}$ 0,9\% segundo a equação 3,4+(0,013 x peso do animal em $\mathrm{kg}$ ) (SEGURA et al., 1997).

Ato contínuo, $10 \mathrm{~mL}$ lidocaina $2 \%$ foi infiltrada na linha vertical do membro pélvico direito e, 
após 25 minutos, a pele, subcutâneo e fáscia muscular foram incisionados e suturados. O membro pélvico esquerdo foi controle e permaneceu íntegro. Este foi T0 e avaliou-se o LNEM. Outras aferições ocorreram a cada 15 minutos até T120 e, então, T240, T360, T480, T720, T1080 e T1440.

As médias dos valores de LNEM obtidos a 1,3 e $5 \mathrm{~cm}$ foram submetidas à análise estatística. Utilizou-se o teste Kruskall Wallis ANOVA em blocos $(\mathrm{P} \leq 0,05)$ para avaliação entre tempos e o teste MannWhitney Rank Sum $(\mathrm{P} \leq 0,05)$ para avaliação entre lados e entre grupos.

\section{RESULTADOS E DISCUSSÃO}

Entre tempos, não houve variação de LNEM nos lados não-incisados de ambos os grupos e também no lado incisado dos animais que receberam amitraz, porém no lado incisado dos animais tratados com xilazina houve redução do LNEM a partir de T480. Entre grupos, no lado incisado de GX verificou-se LNEM inferior ao de GA em T120 e T240, enquanto que no lado não-incisado o LNEM de GX foi menor que o aferido em GA a partir de T120. Entre lados, não houve variação do LNEM nos animais tratados com amitraz, enquanto que nos cavalos que receberam xilazina ocorreu redução do LNEM no lado incisado a partir de T360. Esses dados estão ilustrados na figura 1.

A analgesia promovida pelos agonistas $\alpha^{2}$ é dose-dependente e a redução da transmissão da informação nociceptiva ao córtex cerebral decorre da inibição da liberação de substância P e de noradrenalina observada após a ativação da proteína G (SKARDA \& MUIR III, 1996b; LIN et al., 1998; BUERKLE et al., 1999; LIMA et al., 2000; OLIVERO VÁSQUEZ et al., 2000; GOODRICH et al., 2002). O uso epidural dos agonistas $\alpha^{2}$ pode prolongar seu efeito antinociceptivo (LE BLANC \& CARON, 1990; KEEGAN et al., 1995).

No grupo tratado com xilazina, o LNEM do lado incisado foi similar ao do lado íntegro por mais de quatro e menos de seis horas. Os estudos anteriores descrevem que a injeção epidural de xilazina, em eqüinos, gera antinocicepção por até 220 minutos nos dermátomos inervados pelos nervos caudal, caudal retal, perineal e pudendo (LE BLANC \& EBERHART, 1990; GRUBB et al., 1992; SKARDA \& MUIR III, 1996a; SKARDA \& MUIR III, 1996b; DE ROSSI \& GÓMEZ DE SEGURA, 2001; MUIR III et al., 2001). É provável que o prolongamento da dessensibilização decorra do uso preventivo do fármaco, que é descrito como uma forma eficiente de reduzir a sensibilização central (DANNEMAN, 1997; AIDA et al., 2000; GOODRICH et al., 2002; RÉDUA et al., 2002).
A manutenção da LNEM por mais de 24 horas nos cavalos submetidos ao modelo incisional de dor inflamatória e previamente tratados com injeção epidural de amitraz diluído em emulsão lipídica supera significativamente os valores relatados por VALADÃO (1998), que infere redução da resposta aversiva à estimulação elétrica ou punctória por 120 minutos e aumento da latência frente à estimulação térmica no metatarso por 30 minutos em eqüinos com pele íntegra. Possivelmente, o aumento verificado seja resultante do uso preventivo do amitraz, sugerindo redução da sensibilização central (WOOLF \& BROMLEY, 1999; WARNCKE et al., 2000; PRADO et al., 2002; STANWAY et al., 2002). Além disso, é muito provável que o caráter lipídico do veículo empregado tenha favorecido a permanência do amitraz no interior do canal epidural (FARIAS, 2004; LINARDI, 2004; MENDES, 2004) e, portanto, prolongado a inibição da sensibilização central e a redução dos efeitos deletérios de hipernocicepção.

O volume de lidocaína utilizado foi baixo para evitar espraiamento além da linha incisional e não prolongar a dessensibilização, que é imediata e prolonga-se por 90 minutos (CORTOPASSI et al., 1999; MASSONE, 2002). Como decorreram 30 minutos entre a infiltração de lidocaína e T0 e considerando que a latência para instalação de analgesia após administração epidural, em eqüinos, é de 20 a 45 minutos para xilazina (SKARDA \& MUIR III, 1996b; DE ROSSI \& GÓMEZ DE SEGURA, 2001) e de 30 minutos para o amitraz (VALADÃO, 1998), numa análise mais rigorosa poderia ser sugerido que apenas a partir de T60 o valor de LNEM dependeria exclusivamente dos fármacos administrados pela via epidural. Todavia, adotando-se a mesma metodologia há relato de que a lidocaína $2 \%$ não inibe a hiperalgesia em eqüinos tratados com $\mathrm{NaCl}$ 0,9\% por via epidural (OLESKOVICZ, 2001; RÉDUA et al., 2002).

A metodologia empregada neste estudo foi adequada, pois a incisão cutânea é capaz de induzir dor aguda e hiperalgesia (TVERSKOY et al., 1994). Além disso, a associação deste modelo incisional de dor inflamatória à avaliação do limiar nociceptivo por meio dos filamentos de von Frey já foi utilizada com sucesso em eqüinos (OLESKOVICZ et al., 2001; RÉDUA et al., 2002).

\section{CONCLUSÃO}

Diante do exposto, conclui-se que a injeção preventiva de xilazina ou de amitraz diluído em emulsão lipídica é capaz de manter o limiar nociceptivo à

Ciência Rural, v.39, n.1, jan-fev, 2009. 


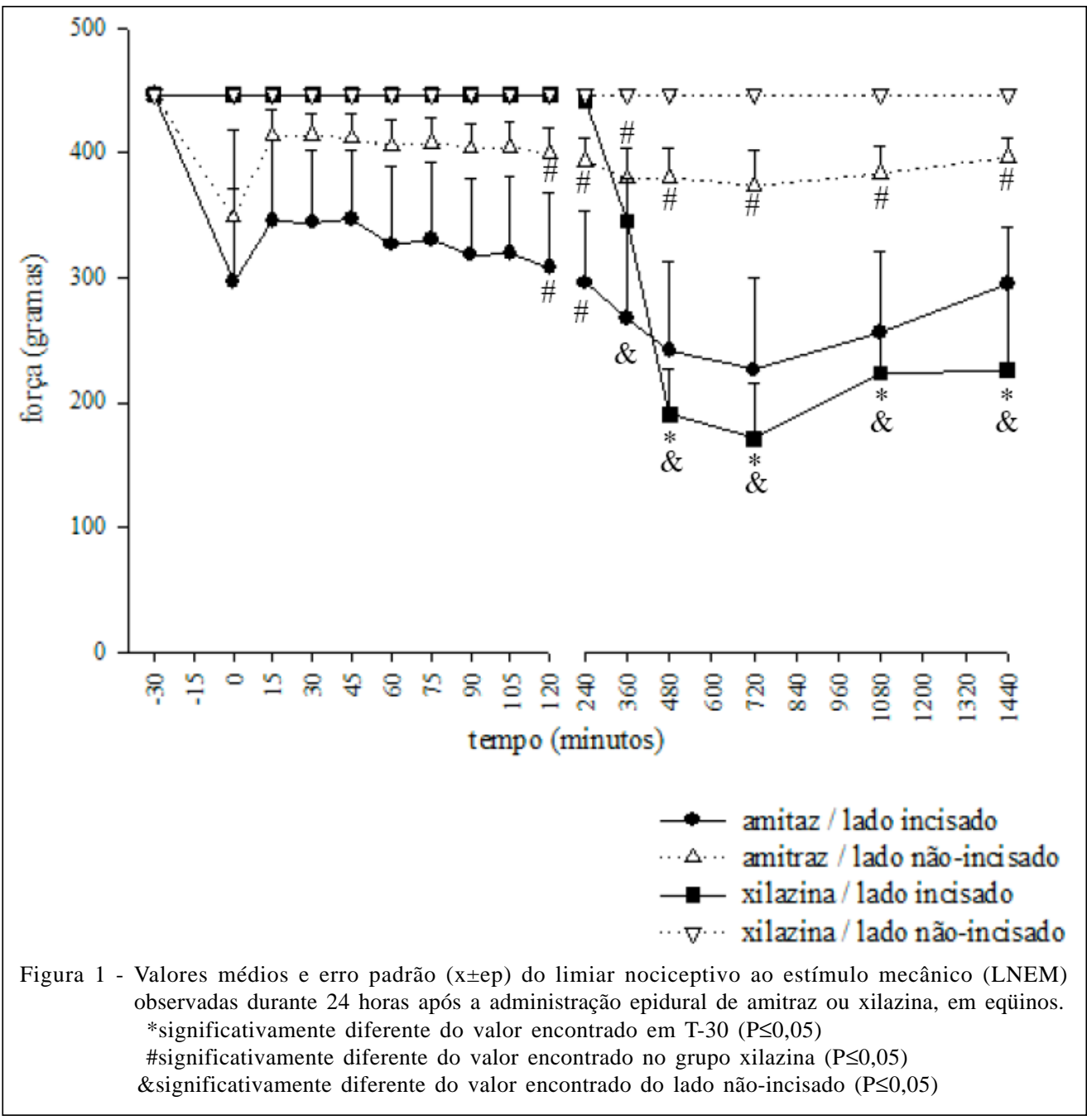

estimulação mecânica por até 4 e 24 horas, respectivamente, em eqüinos submetidos ao modelo incisional de dor inflamatória.

\section{AGRADECIMENTOS}

À Fundação de Amparo a Pesquisa do Estrado de São Paulo - FAPESP (processo $n^{\circ} 03 / 0555-5$ ), pelo apoio financeiro, e à Bayer Saúde Animal pela doação de Rompum ${ }^{\circledR}$.

\section{FONTE DE AQUISIÇÃO}

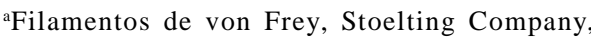
Chicago, Illinois, Estados Unidos.

\section{COMITÊ DE ÉTICA E BIOSSEGURANÇA}

Este trabalho foi conduzido de acordo com as normas éticas após ser aprovado pelo Comitê de Ética e Bem-estar Animal da FCAV - UNESP (protocolo no 009480).

\section{REFERÊNCIAS}

AIDA, S. et al. Preemptive analgesia by intravenous low-dose ketamine and epidural morphine in gastrectomy. Anesth, v.92, n.3, p.1624-1630, 2000.

ALMEIDA, R.M. et al. Avaliação dos efeitos dos amitraz por via epidural em vacas. Braz J Vet Res Anim Sci, v.42, n.6, p.419-428, 2005.

BALESTRERO, L.T. Aspectos farmacológicos da injeção intravenosa ou peridural de amitraz, em eqüinos. 2001. 47f. Dissertação (Mestrado em Cirurgia Veterinária) - Faculdade de Ciências Agrárias e Veterinárias, Universidade Estadual Paulista.

BUERKLE, H. et al. Thermal and mechanical antinociceptive action of spinal vs peripherally administered clonidine in the rat inflamed knee joint model. Br J Anesth, v.83, n.3, p.436441, 1999.

CHEVALIER, H.M. et al. Effect of caudal epidural xylazine on intraoperative distress and post-operative pain in Holstein heifers. Vet Anaesth Analg, v.31, p.1-10, 2004. 
CORTOPASSI, S.R.G. et al. Anestésicos locais. In.: SPINOSA, H.C. et al. Farmacologia aplicada à medicina veterinária. 2.ed. São Paulo: Guanabara Koogan, 1999. Cap.12, p.125130 .

CUNHA, T.M. et al. An electronic pressure-meter nociception paw test for mice. Braz J Med Biol Res, v.37, n.3, p.401407, 2004.

DANNEMAN, P.J. Monitoring of analgesia. In: KOHN, D.F. et al. Anesthesia and analgesia in laboratory animals. London: Academic, 1997. Cap.6, p.83-103.

DE ROSSI, R.; GÓMEZ DE SEGURA, I.A. Efeitos analgésicos, hemodinâmicos e respiratórios da cloridrato de xilazina epidural caudal em eqüinos. Hora Vet, v.120, p.49-53, 2001.

DOHERTY, T.J. et al. Effect of high volume epidural morphine, ketamine and butorphanol on halothane minimum alveolar concentration in ponies. Equine Vet J, v.29, n.5, p.370-373, 1997.

FARIAS, A. Avaliação biotelemétrica e farmacológica da administração sistêmica do amitraz, em cães. 2004. 107f. Tese (Doutorado em Cirurgia Veterinária) - Faculdade de Ciências Agrárias e Veterinárias, Universidade Estadual Paulista, Jaboticabal.

FARIAS, A. et al. Monitoramento continuado de coelhos tratados com amitraz ou xilazina por técnica de biotelemetria. In: COMBRAVET, 29., 2002, Gramado. Anais... Porto Alegre: SOVERGS, 2002. Meio Digital - CD. 2002.

GOODRICH, L.R. et al. Epidural morphine and detomodine decreases postoperative hindlimb lameness in horses after bilateral stifle arthroscopy. Vet Surg, v.31, p.232-239, 2002.

GRUBB, T.L. et al. Comparison of lidocaine, xylazine, and xylazine/lidocaine for caudal epidural analgesia in horses. J Am Vet Med Assoc, v.201, n.8, p.1187-1190, 1992.

GUIRRO, E.C.B.P. Injeção epidural de morfina, xilazina ou clonidina na dor pós-incisional, em eqüinos. 2005. 116f. Dissertação (Mestrado em Cirurgia Veterinária) - Faculdade de Ciências Agrárias e Veterinárias, Universidade Estadual Paulista, Jaboticabal.

HARKINS, J.D. et al. Development and characterization of an equine behaviour chamber and the effects of amitraz and detomidine on spontaneous locomotor activity. J Vet Pharmacol Ther, v.20, p.396-401, 1997.

HSU, W.H. et al. Effect of amitraz on heart and aortic blood pressure in conscious dogs: influence of atropine, prazocin, telazoline and yoimbine. Toxicol Appl Pharmacol, v.84, p.418-422, 1986.

HUGNET, C. et al. Toxicity and kinetics of amitraz in dogs. Am J Vet Res, v.57, n.10, p.1506-1510, 1996.

JONES, S.L. Anatomy of pain. In: SINATRA, R.S. et al. Acute pain - Mechanisms \& management. Missouri: Mosby Year Book, 1992. Cap.2, p.8-28.

KEEGAN, R.D. et al. Cardiovascular effects of epidurally administered morphine and a xylazine-morphine combination in isoflurane-anesthetized dogs. Am J Vet Res, v.56, p.496500, 1995.

LE BLANC, P.H.; EBERHART, S.W. Cardiopulmonary effects of epidurally administered xylazine in the horse. Equine Vet J, v.22, n.6, p.389-391, 1990.

LE BLANC, P.H.; CARON, J.P. Clinical use of epidural xylazine in the horse. Equine Vet J, v.22, n.3, p.180-181, 1990.

LE BLANC, P.H. et al. Epidural injection of xylazine for perineal analgesia in horses. J Am Vet Med Assoc, v.193, n.11, p.1405-1408, 1988.

LEE, I. et al. Antagonistic effects of intravenous or epidural atipamezole on xylazine-induced dorsolumbar epidural analgesia in cattle. Vet J, v.166, p.194-197, 2003.

LEMONICA, L.; PEREIRA, S.M. Dor: bases anátomofisiológicas e do tratamento. In: BRAZ, J.R.C.; CASTIGLIA, Y.M.M. Temas de anestesiologia. Botucatu: Unesp, 1992. Cap.20, p.163-170.

LIN, H.C. et al. Evaluation of analgesia induced by epidural administration of medetomidine to cows. Am J Vet Res, v.59, n.2, p.162-167, 1998.

LINARDI, RL. Efeitos da xilazina e do amitraz sobre as mensurações ecocardiográficas de eqüinos. 2004. 65 f. Dissertação (Mestrado em Cirurgia Veterinária) - Faculdade de Ciências Agrárias e Veterinárias, Universidade Estadual Paulista.

MENDES, M.C. Avaliação do Amitraz e da Romifidina em eqüinos. 2004. 53f. Trabalho de Graduação (Conclusão do Curso de Medicina Veterinária) - Faculdade de Ciências Agrárias e Veterinárias, Universidade Estadual Paulista.

MICHAELIS, M. et al. Silent afferents: a separate class of primary afferents? Clin Exp Pharmacol Physiol, v.23, p.99105, 1996.

MORAES, A.T.B. Lidocaína associada à morfina ou butorfanol, por via epidural, na prevenção da dor pósincisional em éguas. 2003. 64f. Tese (Doutorado em Cirurgia Veterinária) - Faculdade de Ciências Agrárias e Veterinárias, Universidade Estadual Paulista, Jaboticabal.

MUIR III, W.W. et al. Fármacos usados na medicação pré anestésica. In: MUIR III et al. Manual de anestesia veterinária. 3 ed. Porto Alegre: Artmed, 2001. Cap.3, p.3144

OLESKOVICZ, N. et al. Preemptive analgesic effect of epidural ketamine or ketamine $\mathrm{S}(+)$ in postincisional pain in horse. Arch Vet Sci, v.6, p.13, 2001.

OLIVERO VÁSQUEZ, Y.I. et al. Utilidad de la clonidina por via peridural em anestesiología. Rev Med Hosp Gen Mex, v.63, n.4, p.261-266, 2000.

PARADA, C.A. et al. Activation of presynaptic NMDA receptors coupled to NaV1.8-resistant sodium channel C-fibers causes retrograde mechanical nociceptor sensitization. Proc Natl Acad Sci, v.100, n.5, p.2923-2928, 2003.

PRADO, W.A.; PONTES, R.M.C. Presurgical ketoprofen, but not morphine, dipyrone, diclofenac or tenoxican, preempts 
post incisional mechanical allodynia in rats. Braz J Med Biol Res, v.35, n.1, p.111-119, 2002.

QUEIRÓZ-NETO, A. et al. Effect of amitraz and xylazine on some physiological variables of horses. Arq Bras Med Vet Zootec, v.52, n.1, p.27-32, 2000

RÉDUA, M.A. et al. The pre-emptive effect of epidural ketamine on wound sensitivity in horses tested by using von Frey filaments. Vet Anesth Analg, v.29, p.1-7, 2002.

ROBERTS, M.C.; ARGENZIO, A. Effects of amitraz, several opioate derivates and anticholinergic agents on intestinal transit ponies. Equine Vet J, v.18, n.4, p.256-260, 1986.

SALMON, J. et al. L’anesthésie épidurale caudale en pratique equine - aspects anatomiques et techniques. Recuell de Medicine Veterinaire, v.171, p.767-774, 1995

SEGURA, I.G. et al. Epidural injection of ketamine for perineal analgesia in the horse. Vet Surg, v.27, n.4, p.384-391, 1997.

SKARDA, R.T. Local anesthetics and local anesthetic techniques in horses. In: MUIR, W.W.; HOBBELL, J.A.E. Equine anestehesia - Monitoring and emergency therapy. St. Louis: Mosby Year Book, 1991. Cap.10, p.199-246.

SKARDA, R.T.; MUIR III, W.W. Analgesic, hemodynamic, and respiratory effects of caudal epidurally administered xylazine hydrochloride solution in mares. Am J Vet Res, v.57, n.2, p.193-200, 1996a.

SKARDA, R.T.; MUIR III, W.W. Comparison of antinociceptive, cardiovascular, and respiratory effects, head ptosis, and position of pelvic limbs in mares after caudal epidural administration of xylazine and detomidine hydrochloride solution. Am J Vet Res, v.57, n.9, p.1338-1345, 1996b.
STANWAY, G.W. et al. A preliminary investigation comparing pre-operative morphine and buprenorphine for postoperative analgesia and sedation in cats. Vet Anaesth Analg, v.29, p.29-35, 2002.

TVERSKOY, M. et al. Preemptive effect of fentanyl and ketamine on postoperative pain and wound hyperalgesia. Anesth Analg, v.78, p.205-209, 1994.

VALADÃO, C.A.A. et al. Administração epidural de opióides em cães. Cienc Rur, v.32, n.2, p.347-355, 2002.

VALADÃO, C.A.A. et al. Analgesia epidural com xilazina: avaliação cirúrgica e hemogasimétrica. Ars Vet., v.6, n.2, p.125-135, 1990.

VALADÃO, C.A.A. Avaliação da injeção peridural do amitraz em eqüinos. 1998. 63f. Tese (Livre Docência) Faculdade de Ciências Agrárias e Veterinárias, Universidade Estadual Paulista.

WARNCKE, T. et al. Pre-injury treatment with morphine or ketamine inhibits the development of experimentally induced secondary hyperalgesia in man. Pain, v.86, p.293-303, 2000.

WOOLF, C.J.; CHONG, M.S. Preemptive analgesia - treating postoperative pain by preventing the establishment of central sensitization. Anesth Analg, v.77, p.362-379, 1993.

WOOLF, C.; BROMLEY, L. Pre-emptive analgesia by opioids. In: STEIN, C. Opioids in pain control - basic and clinical aspects. Cambridge: Cambridge University, 1999. Cap.11, p.212-233.

WU, C.L. et al. The effect of pain on health-related quality of life in the immediate postoperative period. Anesth Analg, v.97, p.1078-1085, 2003 